\title{
Minimal invasive endoscopic neurosurgery
}

Amit Thapa, Bidur KC, Bikram Shakya

Department of Neurological Surgery, Kathmandu Medical College

Teaching Hospital, Sinamangal, Kathmandu

Correspondence : Dr Amit Thapa, Associate Professor, Department of Neurological Surgery, Kathmandu Medical College Teaching Hospital, Sinamangal, Kathmandu

Email: dramitthapa@yahoo.com

\begin{abstract}
Introduction and Objective: If microscope heralded a new era in delicate and precise neurosurgical procedures, endoscope introduced an element of minimal invasiveness and supplemented microscope where they could not reach. We introduced and have been improving this art in brain and spine surgery and here present our experiences.
\end{abstract}

Materials and Methods: We retrospectively studied patients who underwent endoscopic neurosurgery since July 2014 till January 2016 for brain and spine diseases. Endpoints for the audit were feasibility, reliability, cost effectiveness, extra time taken and morbidity.

Results: We used endoscope in 42 cases. Ventriculoscopy was done for endoscopic third ventriculostomy, septoplasty, cyst aspiration and excision and biopsy. Nasal endoscopy was used to excise pituitary tumor and repair skull base CSF leaks. Spinal endoscopy was performed to remove herniated discs and canal stenosis. Endoscope helped in microscopic excision of acoustic schwannoma as well as craniopharyngioma. We did not have any mortality however CSF leaks were seen which was successfully managed. Since endoscopic procedures involve minimal incision and retraction, patients could be discharged early and mobilised. We discuss our learning phase and limitations of the endoscope with videos and case scenarios.

Conclusion: A continuous phase of learning and problem solving is required to master the art of minimal invasive neurosurgery. Use of endoscope not only supplemented microscope but in cases of ventricular surgery is beyond comparison. It has minimised morbidity as well as hospital stay and is cosmetically acceptable to patients.

Key words: Endoscope, microscope, brain and spine surgery, minimal invasive neurosurgery. 